\title{
Publisher Correction: Real space manifestations of coherent screening in atomic scale Kondo lattices
}

María Moro-Lagares, Richard Korytár, Marten Piantek, Roberto Robles (D), Nicolás Lorente, Jose I. Pascual, M. Ricardo Ibarra \& David Serrate (D)

Correction to: Nature Communications https://doi.org/10.1038/s41467-019-10103-5, published online 17 May 2019.

The original HTML version of this Article was updated shortly after publication because the previous HTML version linked to an incorrect Transparent Peer Review file. The correct Peer Review file has now been linked.

Published online: 13 October 2021

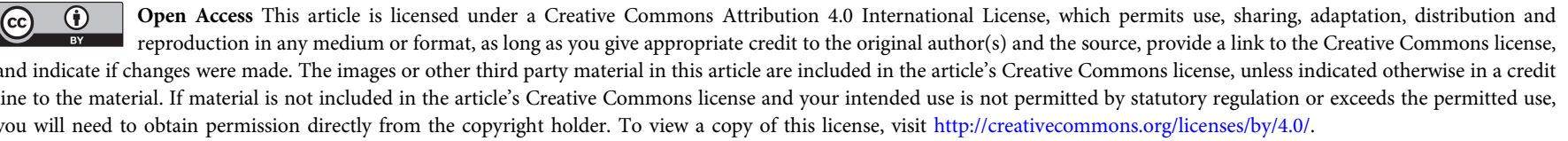

(c) The Author(s) 2021 